\title{
Bone histomorphometry detection of autologous bone powder graft repair of partial mandibular defects in rabbits
}

\author{
J.-Q. Wu ${ }^{1}$, J. Liu ${ }^{1}$, L.-L. Wang ${ }^{1}$, A.-G. Xie ${ }^{1}$ and D.-L. Liu ${ }^{2}$ \\ ${ }^{1}$ Plastic Surgery Department, 463rd Hospital of PLA, Shenyang, China \\ ${ }^{2}$ Department of Plastic and Reconstructive Surgery, \\ Zhujiang Hospital of Southern Medical University, Guangzhou, \\ Guangdong Province, China
}

Corresponding author: J.-Q. Wu

E-mail: jqwucn@126.com

Genet. Mol. Res. 14 (4): 13812-13822 (2015)

Received May 6, 2015

Accepted August 8, 2015

Published October 29, 2015

DOI http://dx.doi.org/10.4238/2015.October.29.1

\begin{abstract}
The aim of this study was to understand the effect of autologous bone powder graft repair of partial mandibular defects of rabbits by the quantitative detection of bone formation. New Zealand rabbits $(\mathrm{N}=18)$ were selected as the test objects, and subjected to bilateral partial mandibular defect induction. One side of the mandibular defect acted as the test group, upon which the autologous bone powder backfilling graft was performed; the other side was put aside and acted as the negative control group. All used an autogenous control. At the twelfth postoperative week, the animals were sacrificed, and semiautomatic image analysis was used to conduct bone histomorphometric detection. Immediately subsequent, quantitative detection of bone formation was performed in the test group. Fluorescent perimeter percent, mineralization apposition rate, and bone formation rate were selected as the dynamic indicators; and trabecular area percent,
\end{abstract}


trabecular thickness, trabecular number, and trabecular separation degree were selected as the static indicators for single factorial variance testing. It was found that the values of $\mathrm{P}$ are less than 0.05 between the test group and the control group, indicating that the effect of autologous bone powder graft repair on partial mandibular defects in rabbits was positive.

Key words: Mandibular defect; Graft; Autologous bone powder; Bone histomorphometry

\section{INTRODUCTION}

The mandible is located at the lower third of the face, bending in a bow shape, and it is one of the bones with the most complex morphology and function in the systemic skeleton. It is also the only bone that can move in the occlusal surface space. The mandible is one of the important factors constituting beauty of the human face; and it also plays an important role in the maintenance of an unobstructed respiratory tract, and in the realization of chewing, swallowing and language functions due to adhesion of the masticatory and suprahyoid muscles. The main causes of mandibular defects include tumor, trauma, and infection, and these and other factors lead to various defects across multiple-anatomic regions. Correspondingly, the respective dysfunctions and requirements of repair and reconstruction differ. The repair of mandibular defects has long been a focus of research in oral plastic surgery (Abu-Serriah et al., 2004; Peled et al., 2005). At present, many methods are used for repairing mandibular defects in China and abroad. Among these, autologous bone grafts (especially bone powder grafts) are one of the more reliable repair options. Autologous bone powder contains live cells, which can be converted into cartilage or osteoblasts to affect osteoacusis (Majumdar et al., 2001; Blum et al., 2004), and it can replace bone formation, induce osteogeny, and directly form bone (Zou et al., 2005). In addition, it has reliable histocompatibility without immunological rejection. Therefore, an autologous bone graft is always used as the "gold standard" for repairing bone defects in the clinic (Babbush, 2001; Mehta and Deschler, 2004; Hurvitz et al., 2006).

Bone histomorphometry, abbreviated as bone metrology, is a branch of biomedical histomorphometry, wherein the parameters of the two-dimensional bone structure are obtained and the bone is reconstructed from sections by means of a bone tissue biopsy, taken according to bone histology and physiology. It is also a quantitative method for describing bone tissue, utilizing stereological techniques to investigate the three-dimensional structure of bone and conduct quantitative observations, and discerning bone tissue morphology and structure on the basis of two-dimensional sections. It can not only provide the objective data of bone formation and bone resorption processes but can also provide the dynamic parameters of bone structure descriptions in the osteon and at the cellular level. From the viewpoint of histocytology, histomorphologic microscopic observations and quantitative analysis of the fracture healing process can accurately reflect the morphology, number, and active degree of a variety of cells participating in the fracture healing process and the rates of bone apposition, mineralization, formation, resorption, and reconstruction by detecting the relevant parameters. Previously, bone metrology was widely used for the detection and testing of osteoporosis (Frost, 1963; Schreurs et al., 2006), functioning as a kind of destruc- 
tive detection method, but was rarely used for detection of bone defect repair and fracture healing. In recent years, however, it has become one of the most effective means for detection of fracture healing. In this study, bone histomorphometry was used to detect the basic structural characteristics and changes of new bone trabeculae in the bone-healing process, and to determine their relationship with the integral structure of bone tissue in order to better understand the subtle dynamic morphological changes in the bone-healing process after autologous bone powder graft.

\section{MATERIAL AND METHODS}

\section{Experimental animals}

Healthy New Zealand rabbits were provided by the Experimental Animal Center of the Affiliated Zhujiang Hospital of Southern Medical University $(\mathrm{N}=18$, male or female, body weight 2.5 to $3.0 \mathrm{~kg}$, average $2.85 \mathrm{~kg}$ ). Rabbits were prepared in accordance with the bilateral mandibular inferior border defect model. One side of the defect was randomly selected as the test group for conducting autologous bone powder backfilling, and the other side, as the "put-aside" defect, was taken as the negative (untreated) control group. All experiments included an autogenous control. This study was carried out in strict accordance with the recommendations in the Guide for the Care and Use of Laboratory Animals of the National Institutes of Health. The animal use protocol was reviewed and approved by the Institutional Animal Care and Use Committee (IACUC) of Plastic Surgery Department of the 463rd Hospital of PLA.

\section{Establishment of the partial mandibular defect model and the graft backfilled with autologous bone powder}

Sumian Lingheji (the animal experiment center of Southern Hospital preparation, 0.3 $\mathrm{mL} / \mathrm{kg}$ ) was administered to subject animals by intramuscular injection for general anesthesia, and the skins in the operation area were prepared and sterilized. Subsequently, surgery was conducted according to the aseptic principle. At the bilateral mandibular inferior borders, skin incisions were designated (incision lengths varied from 3 to $4 \mathrm{~cm}$ ). Skins, subcutaneous tissues, and periost to the mandibular facies ossea were opened and blunt dissection was performed below the periost, with care paid to protecting the nervus mentalis. The masseter muscle adhering to the mandibular body was separated, and bone defects (each defect: $1.0 \mathrm{x}$ $1.0 \mathrm{~cm}$ ) were caused at the bilateral mandibular inferior borders. Sclerotin was ground into bone powder (particle diameter between 50 and $100 \mu \mathrm{m}$ ), and the collected bone powders were backfilled into one side of the defect site, and the other side of defect was put aside. After complete hemostasis, the periost, subcutaneous tissues, and skins were closely sutured. No external dressing was applied. At the wound border, gentamicin $(80,000 \mathrm{U})$ was injected to complete the operation. Subsequently, penicillin (400,000 U; Baiyunshan Pharmaceutical Co., Ltd., Guangzhou Guangdong Province, China) was administered by intramuscular injection daily for 3 days, and wound healing was closely observed. If necessary, timely surgical treatment was conducted. At the 7th postoperative day, the sutures were removed. During the experiment, no animals died accidentally. 


\section{Processing of specimens for bone histomorphometric detection}

Thirteen and fourteen days prior to sacrifice of test subjects, $25 \mathrm{mg} / \mathrm{kg}(0.1 \mathrm{~mL} / 100$ g) tetracycline hydrochloride was administered by hypodermic injection. Three and four days prior to sacrifice, $5 \mathrm{mg} / \mathrm{kg} 0.5 \%$ calcein $(0.1 \mathrm{~mL} / 100 \mathrm{~g})$ was administered by a single hypodermic injection. The time interval between two instances of fluorescence labeling was 10 days. After the 12th postoperative week, six rabbits were killed by the air embolism method. Subsequently, specimen samples were acquired at the left and right extended $0.5 \mathrm{~cm}$ of the junction region of the bone defect and the original bone, and soaked in $10 \%$ formalin-phosphatebuffered solution. For the dynamic and static parameter indicators, the specimens of the 12th postoperative week were used As bone mass was the final measure of bone reconstruction, suggesting the extent of new bone formation, the dynamic and static parameters reflecting bone mass were examined in this study. The dynamic indicators included fluorescent perimeter percent (\% L.Pm), mineralization apposition rate (MAR), and bone formation rate (BFR/ TV). The static indicators included trabecular area percent (\% Tb.Ar), trabecular thickness (Tb.Th), trabecular number (Tb.N), and trabecular separation degree (Tb.Sp). Measurement methodology and calculation formulas were as previously described (Jee et al., 1997). Briefly, the digitizer sketchpad (Summagraphics, Fairfield, CT, USA) and a luminous paintbrush were connected with a light microscope optical system to conduct the semi-automatic image analysis. While imaging, the digitizer sketchpad and vernier screens overlapped with the incision image under the microscope, and the paintbrush moved on the data as if it were moving on the image. Luminous spots on the paintbrush moved along the test tissue structure. Therefore, it was feasible to conduct point counts and measurements of line or curve length, circumference, and area. In addition, detailed programs were set at the border of the digitizer sketchpad screen. According to the test parameters utilized, the corresponding program could be readily selected to conduct measurements, and the measured data could be displayed on the microprocessor connected with the digitizer sketchpad. During the measurement process, it was necessary to conduct random sampling and image acquisition and to maintain accurate records.

\section{Statistical analysis}

Measurement data are reported as means \pm standard deviation (SD), and the SPSS13.0 software (SPSS, Chicago, IL, USA) was used for statistical analysis. If there was no difference between mean values, it was taken as compliance with the factorial variance test. For comparison of mean values, a single factorial variance test was used. Values of $\mathrm{P}<0.05$ were taken as indicating a significant difference.

\section{RESULTS}

\section{Histomorphometric analysis}

The dynamic indicators were: MAR, \% L.Pm, and BFR/TV (Table 1). At the 12th postoperative week, \% L.Pm and BFR/TV values of the test group were significantly greater than those of the control group ( $\mathrm{P}=0.006$ and 0.007 , respectively). The MAR of the test group was also significantly larger than that of the control group $(\mathrm{P}=0.013)$. It was further confirmed that 
the extent of bone mineralization of the test group was higher than that of the control group, suggesting that the effect of the autologous bone powder graft on repair healing was good.

Table 1. Dynamic indices 12 weeks after surgery (means $\pm \mathrm{SD})$.

\begin{tabular}{lcccc}
\hline Group & $\mathrm{N}$ & MAR $(\mu \mathrm{m} /$ day $)$ & $\%$ L.Pm & BFR/TV $(\% /$ year $)$ \\
\hline Control & 6 & $0.867 \pm 0.058$ & $11.757 \pm 0.532$ & $6.200 \pm 0.622$ \\
Experimental & 6 & $1.518 \pm 0.171$ & $18.438 \pm 3.363$ & 4.807 \\
$t$-value & & 8.840 & 12.346 & 9.349 \\
$F$-value & & 9.168 & $0.006^{*}$ & 11.223 \\
P value & & $0.013^{\Delta}$ & $0.007^{*}$ \\
\hline
\end{tabular}

$\mathrm{MAR}=$ mineralization apposition rate; $\% \mathrm{~L} . \mathrm{Pm}=$ fluorescent perimeter percent; $\mathrm{BFR} / \mathrm{TV}=$ bone formation rate; $* \mathrm{P}$ $<0.01 ;{ }^{\Delta} \mathrm{P}<0.05$.

The static indicators are shown in Table 2. At the $12 \mathrm{~h}$ postoperative week, the Tb.Th of the test group was significantly larger than that of the control group $(\mathrm{P}=0.034)$; the $\% \mathrm{~Tb}$.Ar of the test group was also significantly larger than that of the control group $(P=0.016)$, whereas the Tb.Sp of the test group was significantly lower than that of the control group $(\mathrm{P}=0.010)$. In addition, the Tb.N value of the test group was greater than that of the control group $(\mathrm{P}=0.001)$. These results suggested that there was frequent bone trabecula formation in the test group, with a low degree of separation, indicating that bone trabeculae were widely distributed.

\begin{tabular}{|c|c|c|c|c|c|}
\hline Group & $\mathrm{N}$ & $\% \mathrm{~Tb} . \mathrm{Ar}$ & $\mathrm{Tb} . \mathrm{Sp}(\mu \mathrm{m})$ & Tb.N $(\mu \mathrm{m})$ & Tb.Th $(\mu \mathrm{m})$ \\
\hline Control & 6 & $13.501 \pm 0.208$ & $867.122 \pm 24.683$ & $998 \pm 28$ & $135.316 \pm 3.444$ \\
\hline Experimental & 6 & $23.090 \pm 0.663$ & $537.695 \pm 55.862$ & $1444.3 \pm 146$ & $161.337 \pm 16.666$ \\
\hline$t$-value & & 33.797 & -13.213 & 7.350 & 3.745 \\
\hline$F$-value & & 8.364 & 10.038 & 20.438 & 6.045 \\
\hline$P$ value & & $0.016^{\Delta}$ & $0.010^{\Delta}$ & $0.001 *$ & $0.034^{\Delta}$ \\
\hline
\end{tabular}

$\% \mathrm{~Tb} . \mathrm{Ar}=$ trabecular area percent; $\mathrm{Tb} . \mathrm{Sp}=$ trabecular separation degree; $\mathrm{Tb} . \mathrm{N}=$ trabecular number; $\mathrm{Tb} . \mathrm{Th}=$ trabecular thickness. $* \mathrm{P}<0.01 ;{ }^{\Delta} \mathrm{P}<0.05$.

\section{Masson-Goldner trichrome staining of sections}

After Masson-Goldner trichrome staining of sections, the electronic microscope was used for observation. It was found that the non-mineralized osteoid was dyed red and the sclerotin was green, osteocytes were located in the bone trabecular, the nucleus was dyed dusty blue, and the cytolymph was also dyed red. For the test group, abundant sclerotins could be observed. The bone trabeculae were widely distributed and had good morphologies. In addition, numerous osteocytes were distributed between the bone trabeculae. In the control group, there were many osteoid components remaining, whereas there were fewer osteocytes and the bone trabeculae were sparsely distributed (Figures 1 and 2).

\section{Von Kossa staining of sections}

Following Von Kossa staining of sections, observations were conducted under fluorescence microscopy. Calcein deposition on the bone surface was stained green, and tetracycline fluorescence deposition appeared yellow. The labeled sclerotin mineralization apposition 
could be visualized between the two fluorescence bands, indicating sclerotin mineralization apposition rate (Figures 3 and 4).

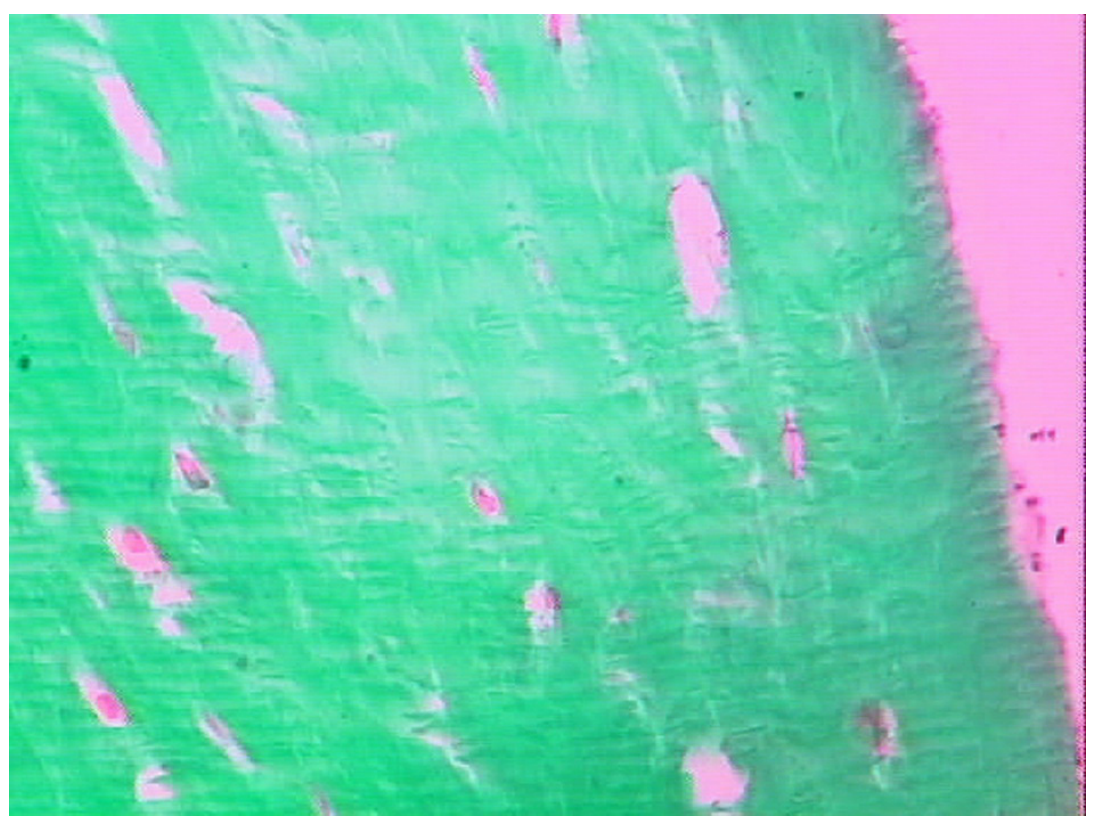

Figure 1. Experimental group at the 12th postoperative week. Masson-Goldner stain (400X).

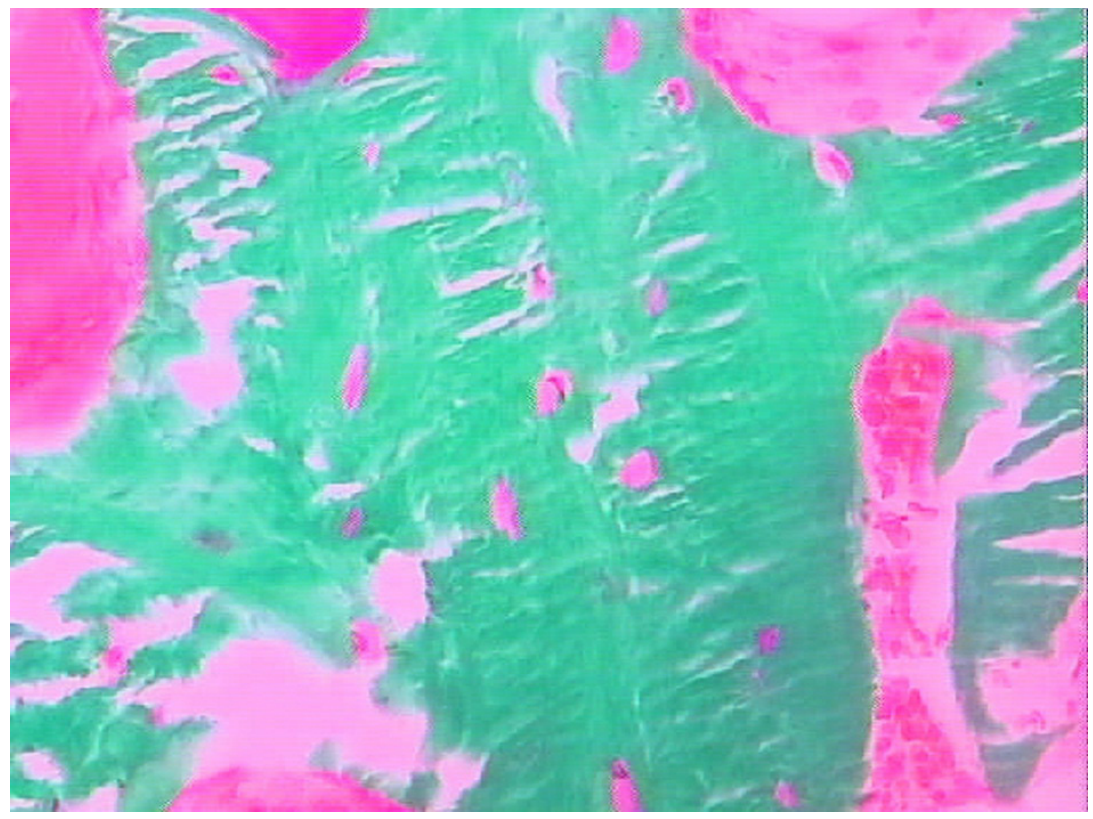

Figure 2. Negative control group at the 12th postoperative week. Masson-Goldner stain (400X). 


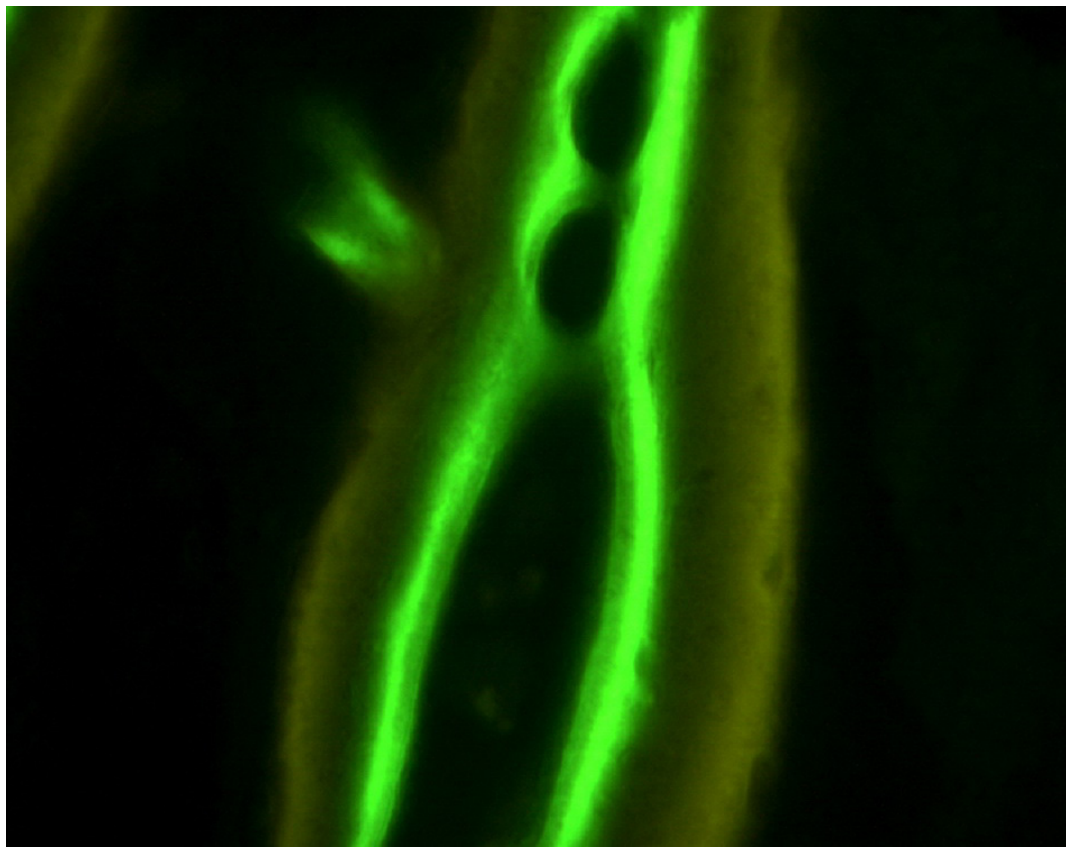

Figure 3. Experimental group at the 12th postoperative week. Von Kossa stain; fluorescence microscope (200X).

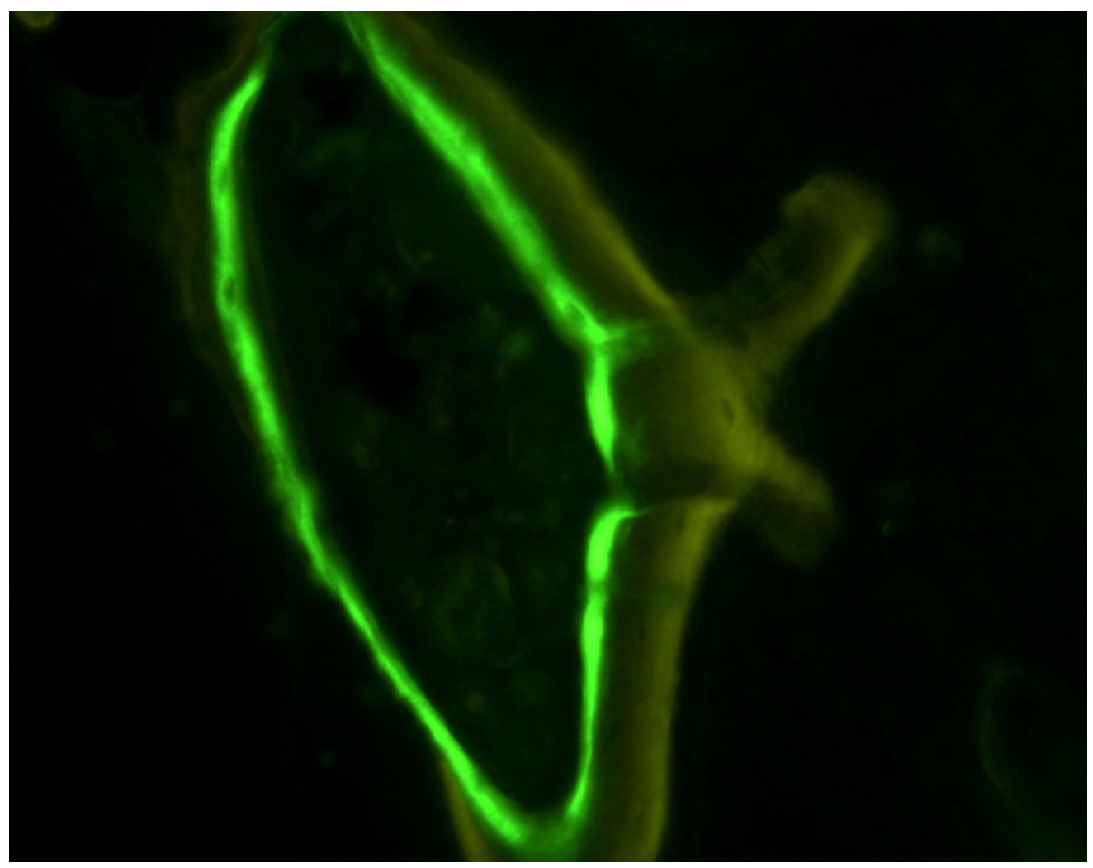

Figure 4. Negative control group at the 12th postoperative week. Von Kossa stain; fluorescence microscope (200X). 


\section{DISCUSSION}

Partial mandibular defects can cause severe facial deformities in patients, and can even cause lingual and masticatory dysfunctions (Sclaroff et al., 1994). Therefore, the repair and reconstruction of mandibular defects is one of the most difficult clinical problems in the head and neck surgery reconstruction field, because it is necessary to not only repair the mandibular continuity and facial appearance but also, and more importantly, to restore functionality to patients to improve quality of life. Autologous bone powder is derived from a patient's own tissue, and has regenerative repair capacity. As it harbors a number of bonegrowth-stimulating proteins, it can promote bone induction (Springfield, 1996), and is the bone graft material with the most potential osteogenic activity in the body. After grafting, it rapidly fuses with bone receptors. In addition, immunologic rejection rarely occurs, and there are few complications (Wang et al., 2005). Additional advantages for use of autologous bone powders in grafts include a fast creeping substitution process, high strength of the formed callus, and short bone defect repair time (Arlet et al., 2006). For a deeper understanding of the osteogenic mechanisms of autologous bone powder, we used the platform of an animal model of autologous bone powder mandibular defect repair, combined with bone histomorphometric assay methodologies to detect the basic structural characteristics and changes of new bone trabeculae in the bone-healing process, and their relationship with the integral structure of bone tissue. We further utilized a tetracycline live-bone tissue fluorescence-labeling technique to indirectly detect new bone-formation rates to further understand the subtle morphological dynamic changes of the bone-healing process after autologous bone powder grafts. The results of this study showed that autologous bone powder graft repair of partial mandibular defects carried advantages over other techniques, such as a short osteogenic time, good morphology, and reliable osteogeny.

Traditional bone tissue detection methods include nondestructive detection by ultrasonic waves, $\mathrm{X}$-rays, and computed tomography and bone density scans, and the destructive detection using specimens and tissue sections. These detection means can only reflect the changes of bone morphology or quality, but cannot quantitatively describe the specific changes of bone mass. Bone histomorphometry is one method for quantitative examination of nondecalcified hard tissue sections. It can provide quantitative information on bone reconstruction and bone structure and can provide the parameters of three-dimensional bone structure and reconstruction. For the acquisition and evaluation of information on bone metabolism, bone histomorphometry can be used to conduct unique and more complete descriptive analyses compared to other methods. Although it belongs to the category of destructive detection, it can detect the formation and process changes of bone tissue with greater accuracy. Therefore, it has wide application value in etiopathogenesis research, drug efficacy evaluation, clinical osteonosus research, clinical drug research and development, and other fields (Weinsten, 2008). Based on these advantages, we designed bone histomorphometry detection indicators specific for understanding the subtle, dynamic morphological changes of the bone-healing process after autologous bone powder graft.

Bone histomorphometry parameters can be divided into two classes: dynamic and static parameters (Dempster et al., 1993). Dynamic parameters provide indication of the bone formation rate. Tetracycline can be used as a marker to clearly observe the dynamic changes of sclerotin apposition in the bone regeneration process. The ability of bone to specifically com- 
bine with tetracycline and calcein for deposition on the bone mineralization front can be used to label the time factor in the bone regeneration and reconstruction process; the mineralization band of sclerotin apposition occurs between them. Under the fluorescence microscope, sites of specific staining are observed and measured. Together, these observations allow the dynamic parameters of bone tissue to be determined (Parfitt, 1983; Salle et al., 2002). The parameters reflecting bone formation include MAR, the rate of mineralization layer formation on the bone trabecula surface (the distance between the double markers is divided by the time interval between markers to obtain the MAR); \% L.Pm, representing the formation amount on the mineralized bone surface; and BFR/TV, the new bone mass on unit bone surface in units of time as the result of the mineralization surface multiplied by the mineralization apposition rate. Among the dynamic parameters, \% L.Pm reflects the number of osteoblasts, MAR reflects the functional status of which osteoblast is mineralization osteoid, while BFR/TV reflects the comprehensive situation of osteoblast number, osteoid morphology, and mineralization, and it also reflects the comprehensive situation of bone formation as a whole. In addition, MAR and BFR/TV can dynamically show osteoblast changes and the extent of bone mineralization in the osteogenic process, showing a series of bone formation processes. In this experiment, the MAR of the test group was significantly larger than that of the negative control group $(\mathrm{P}=$ $0.006)$, and $\mathrm{BFR} / \mathrm{TV}$ was further and significantly greater than that of the control group $(\mathrm{P}=$ 0.007); both results suggested that bone formation in the test group was considerably greater than that in the control group. In addition, electron and fluorescence microscope observations also showed that osteogeny of the test group was active, and that the test group morphology and distribution of bone trabeculae were better than those of the control group. Further, the osteoid of the test group was smaller than that of the control group. Together, these findings suggest that autologous bone powder is a suitable graft for repairing mandibular defects, wherein it can markedly increase the rate of sclerotin mineralization and promote bone healing.

Static parameters include $\mathrm{Tb}$.Th, referring to average trabecular thickness; \% $\mathrm{Tb}$.Ar, reflecting the amount of bone mass, whose change can influence bone mass; and Tb.N, referring to the number of trabeculae in a unit area. In the condition of constant Tb.N, the thicker the $\mathrm{Tb} . \mathrm{Th}$, the greater the \% Tb.Ar; in the condition of constant Tb.Th, the greater the Tb.N, the greater the $\% \mathrm{~Tb}$.Ar. Another static parameter is the Tb.Sp, referring to the average distance between two adjacent bone trabeculae. The larger the Tb.Sp, the larger the distance between bone trabeculae, and sclerotin is looser. These parameters quantitatively reflect the morphological structures of bone trabeculae, and describe in detail the changes of bone trabeculae in the generation of new bone and in the reconstruction process in bone regeneration. Among these, $\mathrm{Tb} . \mathrm{Th}, \% \mathrm{~Tb} . \mathrm{Ar}$, and $\mathrm{Tb} . \mathrm{N}$ show the amount of bone trabeculae in the bone regeneration process. Larger values indicate more active bone trabecula formation and greater bone mass. However, Tb.Sp represents the amount and maturation extent of bone trabeculae. If its value is larger, it suggests that the bone trabeculae are rare, and that osteogenic differentiation is poor. In this study, the Tb.N of the test group was significantly larger than that of the negative control group $(\mathrm{P}=0.001)$, and $\mathrm{Tb}$.Th and $\% \mathrm{~Tb}$.Ar were significantly larger than those of the negative control group $(\mathrm{P}=0.034$ and 0.016 , respectively). The Tb.Sp of the test group was significantly lower than that of the control group $(\mathrm{P}=0.010)$, suggesting that autologous bone powder promoted trabecular formation and differentiation, widely distributed bone trabeculae to quicken bone healing, good bone reconstruction morphology, and reliable osteogeny, in line with previous findings (Heiner et al., 2007). 
Although we can understand the subtle morphological changes of new bone tissue in the bone-healing process after autologous bone powder graft by bone histomorphometry detection, this method has some shortcomings, such as difficult sampling, difficult hard tissue section preparation, and extended research time. Furthermore, this detection method is destructive (Parfitt et al., 1987; Barger-Lux and Recker, 2002), and will therefore cause a new trauma to the body. Therefore, its application has certain limitations. In recent years, although allogeneic bone and xenogeneic bone grafts and various tissue engineering bone materials processed by different methods have been developed, autologous bone powder graft is the most commonly used method in mandibular defect repair, and its success rate is the highest (Matsumura and Kawasaki, 2000). Autologous bone powder graft repair of partial mandibular defects meets desired physiological requirements, and its histocompatibility is reliable. It acts as a good osteoacusis stent and carries the advantages of reliable osteogeny, rapid healing, and obvious efficiency. It utilizes a bone graft material with the greatest osteogenic activity, and its clinical application continues to increase.

Bone histomorphometry can provide the parameters of three-dimensional bone structure and reconstruction and can conduct quantitative evaluation and provide bone metabolism information. It is a powerful technique that is incomparable with any other methodology. Furthermore, it can more intuitively conduct the quantitative detection of bone histomorphometry indicators of bone diseases, and dynamically describe the bone-healing process. Therefore, it elevates bone formation detection in bone defect repair at the quantitative, dynamic, and microcosmic extent, and lays a preliminary experimental basis for the clinical application of autologous bone powder repair of partial mandibular defects.

\section{REFERENCES}

Abu-Serriah MM, Odell E, Lock C, Gillar A, et al. (2004). Histological assessment of bioengineered new bone in repairing osteoperiosteal mandibular defects in sheep using recombinant human bone morphogenetic protein-7. Br. J. Oral. Maxillofac. Surg. 42: 410-418.

Arlet V, Jiang L, Steffen T, Ouellet J, et al. (2006). Harvesting local cylinder autograft from adjacent vertebral body for anterior lumbar interbody fusion: surgical technique, operative feasibility and preliminary clinical results. Eur. Spine J. 15: 1352-1359.

Babbush CA (2001). Bone: Present and future. In: Dental implants. The Art and Science. W.B. Saunders Company, Philadephia, 59-82.

Barger-Lux MJ and Recker RR (2002). Bone microstructure in osteoporosis: transilial biopsy and histomorphometry. Top. Magn. Reson. Imaging 13: 297-305.

Blum B, Moseley J, Miller L, Richelsoph K, et al. (2004). Measurement of bone morphogenetic proteins and other growth factors in demineralized bone matrix. Orthopedics 27: S161-165.

Dempster DW, Ferguson-Pell MW, Mellish RW, Cochran GV, et al. (1993). Relationships between bone structure in the iliac crest and bone structure and strength in the lumbar spine. Osteoporos. Int. 3: 90-96.

Frost HM (1963). Measurement of human bone formation by means of tetracycline labeling. Can. J. Biochem. Physiol. 41: 31-42.

Heiner AD, Callaghan JJ and Brown TD (2007). Stability of fused versus nonfused THA femoral impaction grafts. $J$. Orthop. Res. 25: 351-360.

Hurvitz KA, Kobayashi M and Evans GR (2006). Current options in head and neck reconstruction. Plast. Reconstr. Surg. 118: 122e-133e.

Jee WS, Li XJ and Inoue J (1997). Histomorphometric assay of growing long bone. In: Handbook of Bone Morphometry. 2nd edn. (Takehashi H, ed.). Nishimura Shoten, Niigata, 87-110.

Majumdar MK, Wang E and Morris EA (2001). BMP-2 and BMP-9 promotes chondrogenic differentiation of human multipotential mesenchymal cells and overcomes the inhibitory effect of IL-1. J. Cell Physiol. 189: 275-284. 
Matsumura H and Kawasaki K (2000). Magnetically connected removable sectional denture for a maxillary defect with severe undercut: a clinical report. J. Prosthet. Dent. 84: 22-26.

Mehta RP and Deschler DG (2004). Mandibular reconstruction in 2004: an analysis of different techniques. Curr. Opin. Otolaryngol. Head Neck Surg. 12: 288-293.

Parfitt AM (1983). The physiologic and clinical significance of bone histomorphometric data. In: Bone Histomorphometry: Techniques and Interpretation (Recker RR, ed.). CRC Press, Boca Raton, 143-223.

Parfitt AM, Drezner MK, Glorieux FH, Kanis JA, et al. (1987). Bone histomorphometry: standardization of nomenclature, symbols, and units. Report of the ASBMR Histomorphometry Nomenclature Committee. J. Bone. Miner. Res. 2: 595-610.

Peled M, El-Naaj IA, Lipin Y and Ardekian L (2005). The use of free fibular flap for functional mandibular reconstruction. J. Oral. Maxillofac. Surg. 63: 220-224.

Salle BL, Rauch F, Travers R, Bouvier R, et al. (2002). Human fetal bone development: histomorphometric evaluation of the proximal femoral metaphysis. Bone 30: 823-828.

Schreurs BW, Arts JJ, Verdonschot N, Buma P, et al. (2006). Femoral component revision with use of impaction bonegrafting and a cemented polished stem. Surgical technique. J. Bone Joint Surg. Am. 88: 259-274.

Sclaroff A, Haughey B, Gay WD and Paniello R (1994). Immediate mandibular reconstruction and placement of dental implants. At the time of ablative surgery. Oral Surg. Oral Med. Oral Pathol. 78: 711-717.

Springfield D (1996). Autograft reconstructions. Orthop. Clin. North Am. 27: 483-492.

Wang YJ, Tong YQ, Dai Q, Chen AG, et al. (2005). The influence of autogenous bone graft in cleft palate on maxillary growth: an experiment study in rats. Shanghai Kou Qiang Yi Xue 14: 37-41.

Weinsten R (2008). Clinical use of bone densitometry. In: Osteoporosis. 3rd edn. (Marcus R, Feldman D, Nelson D and Rosen CJ, eds.). Academic Press, San Diego, 448-465.

Zou T, Yan J, Fu H, Hou Z, et al. (2005). Combined use of autologous micro-morselized bone with bone morphogenetic protein and type I collagen graft in repairing rabbit bone defects. Zhongguo Xiu Fu Chong Jian Wai Ke Za Zhi 19: $187-191$. 